Inken Keim

\title{
Städtische Gruppen und ihre kommunikativen sozialen Stile
}

\begin{abstract}
In der Chicago Schule der Soziologie wurde in der Tradition des symbolischen Interaktionismus das Konzept der „sozialen Welt“ entwickelt und zur Erfassung von städtischen Lebenswelten und Subkulturen verwendet. Soziale Welten haben eine gemeinsame Kultur mit verbindlichen Wissensbeständen und Ausdruckssystemen, die sich als ökonomisch und vorbildhaft für den Erfolg von Handlungsdurchführungen erweisen. Zur Bearbeitung von Aufgaben können sich innerhalb von sozialen Welten lokal verankerte Gruppen bilden, die für die ethnografische Beobachtung gut geeignet sind. Zur Erfassung der in solchen Gruppen ausgebildeten Sprachund Kommunikationsweisen erweist sich das Konzept des kommunikativen Stils als geeignetes Instrument.

Nach Darstellung des theoretischen Ansatzes und empirischen Vorgehens werden verschiedene Gruppen vorgestellt, ihre kommunikativen Stile charakterisiert und durch Vergleich sozial differenzierende Merkmale aufgezeigt: Eine Gruppe von Frauen aus der „Welt der kleinen Leute“ im Vergleich zu einer Frauengruppe aus dem Bildungsbürgertum; und eine Gruppe junger Deutschtürkinnen, deren kommunikativer Stil sich im Laufe ihrer biografisch-sozialen Entwicklung verändert hat.
\end{abstract}

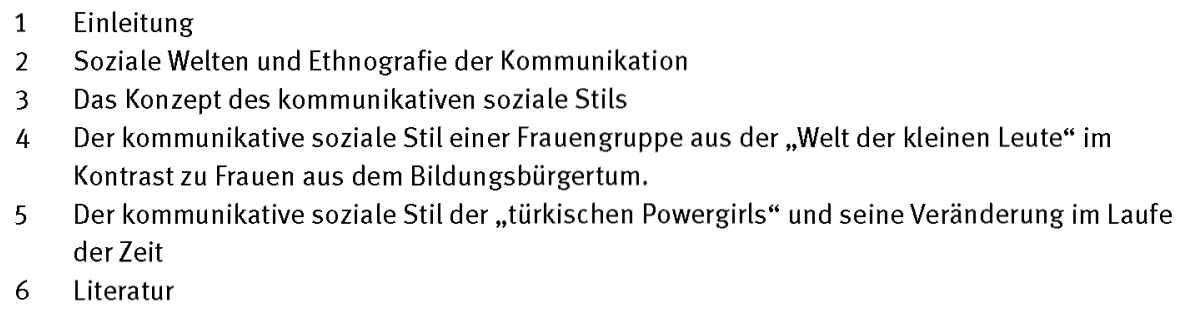

\section{Einleitung}

Theoretische Ansätze und empirische Untersuchungen zur sozialen Organisation des Lebens in der Stadt entstanden bereits in den 20er und 30er Jahren des 20.Jhds. in der Chicago-Schule der Soziologie. Als Untersuchungseinheiten wurden stadttypische Wohnquartiere ausgewählt, die durch enge nachbarschaftliche Beziehungen charakterisiert waren. Dabei handelte es sich um städtische Teilgebiete, die durch die Mechanismen sozialer Segregation und Ausdifferenzierung im Zuge der Arbeitsteilung zwischen Wohnen und Arbeiten, Arbeiten und Freizeit, der Differenzierung zwischen Alteingesessenen und Zuwanderern ebenso wie durch infrastrukturelle und 
bauliche Um- und Neugestaltungen entstanden sind und hinsichtlich bestimmter ethnischer und sozial-ökonomischer Merkmale eine gewisse Homogenität aufweisen. Die Stadtviertelforschung hatte vor allem in Einwanderervierteln enge sozial-ökologische Strukturen festgestellt, die im Kontrast zur Urbanität städtischer Zentren standen. Die Untersuchungen von engen Nachbarschaften (z. B. Suttles 1968), von jugendlichen Gruppen oder Banden (z. B. Whyte 1943) oder von Stätten des Freizeitvergnügens (Cressey 1932/1969) zeigten die Gliederung der städtischen Population in kleinere Subwelten mit engem sozialem Zusammenhalt und eigenen Subkulturen. Als Basis für die soziolinguistische und sozialstilistische Erfassung und Beschreibung städtischer Subwelten und ihrer charakteristischen Sprach- und Kommunikationsformen, das zeigt diese frühe Forschung, eignen sich die Konzepte „soziale Welt“ und „Ethnografie der Kommunikation“ in besonderer Weise.

\section{Soziale Welten und Ethnografie der Kommunikation}

Nach Anselm Strauss (1984) sind soziale Welten dynamische Gebilde; sie haben die Tendenz zur Ausdifferenzierung in Subwelten und zur Verzahnung mit anderen sozialen Welten. Im Vergleich zu soziologischen Ansätzen, die von relativ klar umrissenen sozialstrukturellen Einheiten wie Nation, Klasse, Ethnie, Institution, Geschlecht, Familie etc. ausgehen, ist Strauss‘ Konzept der sozialen Welt sehr offen; soziale Welten können klein oder groß sein, lokal gebunden oder trans-/oder international organisiert sein, feste oder sehr durchlässige Grenzen haben, ethnisch, geschlechtlich und altersmäßig homogen oder gemischt sein und Menschen aus unterschiedlichen sozialen Schichten vereinen. Kennzeichnend für eine soziale Welt ist die Fokussierung auf Kernaktivitäten, mit denen sich alle zur sozialen Welt Gehörenden identifizieren und die sie gemeinsam bearbeiten. Das erfolgt in einem situationsübergreifenden Arbeitszusammenhang und führt zum Aufbau von Netzwerken zur Beschaffung von Ressourcen und zur Rekrutierung von Mitarbeitern. Soziale Welten haben eine „gemeinsame Kultur mit Wir-Bewusstsein“ (Schütze 2002, 63) und verbindliche Wissensbestände. Es muss immer wieder entschieden werden, was eigene, authentische Handlungen und Durchführungsweisen sind. So entstehen Kommunikationsweisen und Verhaltensstile, die sich als ökonomisch und vorbildhaft für den Erfolg der Handlungsdurchführung erweisen. Sie gelten als Leitbilder für die Angehörigen und werden zum Vorbild für neue Mitarbeiter.

Innerhalb von sozialen Welten können sich zur Bearbeitung spezifischer Aufgaben soziale Gruppen bilden, z. B. politische, sportliche oder Freizeitgruppen. In solchen Gruppen finden Debatten über die Lösung von Aufgaben statt, die für die Ausbildung von Kommunikationspraktiken von zentraler Bedeutung sind. Hier werden explizite Bewertungen und Definitionen von Ausdrucksformen vorgenom- 
men und symbolisierende Verfahren praktiziert, die die Teilnehmer als ,zu uns“ oder „nicht zu uns gehörig“ bestimmen. Den Bezugsrahmen für Gruppendebatten bildet nicht die Identität der Gruppe als stabile Einheit, sondern die soziale Welt, in deren Handlungszusammenhang die Gruppenbildung und die Definition der relevanten Sozialbeziehungen stattfinden. Wenn Gruppen lokal verankert sind, sind sie für die ethnografische Beobachtung und Beschreibung besonders gut geeignet.

Die Ethnografie der Kommunikation ist eine geeignete Methode zur Erfassung der sozial- kulturellen Zusammenhänge, in denen Interaktionsbeteiligte leben, und in denen sie die Wissensbestände erwerben, auf die sie bei der interaktiven Bedeutungsherstellung rekurrieren. Die Ethnografie der Kommunikation wurde von Gumperz und Hymes (1964, 1972) als Basis der Soziolinguistik etabliert. Mit dem ethnografischen Ansatz ist es möglich, auf der Mikro- und Meso-Ebene gesellschaftlicher Organisation durch Beobachtung und Dokumentation des Miteinander-Sprechens von Mitgliedern einer Gemeinschaft die soziale Konstruktion von Wirklichkeit zu erfassen und zu beschreiben. In der Art und Weise, wie Mitglieder miteinander und übereinander sprechen, werden die für die Gemeinschaft geltenden Normen und Wertorientierungen ausgehandelt und die relevanten sozialen Kategorien und Identitäten ausgearbeitet; es werden die Funktionen verwendeter Sprachen und Sprechweisen, bevorzugte Thematisierungen, ebenso wie Gesprächsregeln für Lob und Kritik deutlich. Das ethnografische Vorgehen liefert ein weites Kontextwissen über die beobachtete Gemeinschaft durch teilnehmende Beobachtung und Audio- oder Videodokumentation von sozialen Ereignissen, durch Interviews mit Mitgliedern und durch Daten von Außenstehenden über die Gemeinschaft. Die Aufgabe ethnografischer Untersuchungen ist es, soziale Welten und ethnografisch repräsentative Gruppen sozialer Welten zu identifizieren, Schüsselsituationen der Kommunikation festzulegen und insgesamt den Rahmen für die Analyse von kommunikativen sozialen Stilen zu liefern. (Kallmeyer 1995a).

\section{Das Konzept des kommunikativen sozialen Stils}

In der linguistischen Stilforschung wird mit Stil der Zusammenhang von sehr unterschiedlichen Ausdrucksmitteln bezeichnet, die auf verschiedenen Ausdrucksebenen über längere Strecken hinweg erscheinen. In dieser Forschungstradition wird stilistische Variation als Wahl zwischen zwei oder mehr bedeutungsähnlichen Ausdrucksalternativen verstanden, die den Arten der Handlungsdurchführung differenzierenden sozialen Wert verleiht. Nach dem Konzept des kommunikativen sozialen Stils, das in den Mannheimer Stadtprojekten entwickelt wurde (vgl. Kallmeyer 1995b, Keim 1995a und Schwitalla 1995a) haben Sprecher jedoch keine Wahl zwischen Alternativen, wenn sie ihre Zugehörigkeit zu einer bestimmten sozialen Welt ausdrücken wollen, sondern der in dieser Welt ausgeprägte Stil ist Ausdruck ihrer sozialen und kultu- 
rellen Identität. Dieses Konzept des kommunikativen sozialen Stils knüpft an ethnografische (z. B. Heath 1983), soziologische (z. B. Soeffner 1986), anthropologische (z. B. Irvine 2001) Ansätze an, die auf die Untersuchung von kulturellen Stilen ausgerichtet sind, an das Stilkonzept der „cultural studies“ (Clarke 1979, Willis 1981) und an den kultursoziologischen Ansatz Bourdieus (1982) zur sozialen Differenzierung der Gesellschaft. Kommunikative soziale Stile, in denen sich gesellschaftliche und interaktive Bedeutungen verbinden, bilden die Brücke zwischen lokal stattfindenden Interaktionen und den in übergreifenden Sozialwelten gebundenen kulturellen Traditionen, Überzeugungen und Wissensbeständen.

In dem Konzept des kommunikativen sozialen Stils ist Stil sozial-funktional definiert. Sprecher setzen Stilformen als Mittel zum Ausdruck sozial-kultureller Zugehörigkeit und zur sozialen Positionierung in Relation zu relevanten Anderen ein. Stil bildet im Sinne von Bourdieu (1982) das Kapital für die Auseinandersetzung mit anderen sozialen Welten. Stil wird holistisch als Gestalt-Konzept gefasst, in dem Elemente auf allen Ausdrucksebenen in homologer Weise miteinander kombiniert und sprachliche Ausdrucksmittel mit Merkmalen der Aufmachung und Kleidung, des Körperverhaltens und insgesamt der geschmacklichen Ausrichtung „gleichsinnig“ und einer zentralen „Logik“ folgend zu einer einheitlichen Gestalt, zu einem Bild, einer Figur bzw. einem Hyperzeichen formiert werden. Stile drücken die Orientierung an sozialen Leitbildern aus, an denen Interaktanten ihr kommunikatives Handeln ausrichten.

Stile sind prototypisch organisiert, werden um Kern- bzw. Leitphänomene herum aufgebaut und haben unscharfe Grenzen. Sie setzen ein gewisses Maß an Konventionalisierung voraus, für die es Bezeichnungen gibt und die mit bestimmten sozialen Einheiten assoziiert werden (Soeffner 1986, Willis 1981). Stile werden unter Aspekten der ästhetischen Performanz mit dem Ziel der Hochstufung sozialer Differenz entwickelt. Dazu werden besonders die Merkmale hervorgehoben, die stark mit denen eines anderen sozialen Stils kontrastieren. Stile haben für Gesprächsbeteiligte ebenso wie für Analysierende Kontextualisierungsfunktion. Durch die Verwendung eines bestimmten Stils oder durch Stilwechsel können die Interaktanten unterschiedliche Kontexte herstellen, in denen dieser Stil Relevanz hat.

Stile werden interaktiv hergestellt, d. h. Sprecher und Rezipienten nehmen an der Herausbildung von Stilen ebenso wie an ihrer Aufrechterhaltung und Veränderung teil. Stile umfassen alltägliche Handlungsweisen, die für die Gesellschaftsmitglieder fraglos gegeben sind, z. B. Routinen, die an einer Formulierungsmodalität der Selbstverständlichkeit erkennbar sind. Die routinierten Handlungsweisen verschaffen die Möglichkeit, dass Teilnehmer sich als Gleichgesinnte wahrnehmen und durch schnellen Austausch von sich ergänzenden Äußerungen sich wechselseitig signalisieren, dass sie in ihrer eigenen Welt sind. Neben Routinen gibt es auch hervorgehobene Stilisierungen, in denen Merkmale in besonderer Weise überhöht werden. Das geschieht vor allem bei Abgrenzungshandlungen, bei Stildiskussionen und bei Kritik am Verhalten anderer. 
Die Verknüpfung von Konversationsanalyse und Kontextualisierungskonzept liefert das Instrumentarium für die Analyse der lokalen Herstellung von Bedeutung durch Stile in Interaktionen. Zur Beschreibung des kommunikativen Stils sozialer Gruppen haben sich vor allem die folgenden Stildimensionen als relevant erwiesen:

- der Umgang untereinander und mit Außenstehenden: Hier spielen Regeln für den Umgang mit Territorien, für Thematisierungen, für Lob und Kritik, ebenso wie für die Herstellung von Gemeinsamkeit eine Rolle;

- der Einsatz von Sprachen, Varietäten und Sprechweisen zur Interaktionsorganisation und zur Symbolisierung sozialer Eigenschaften;

- die Ausbildung eines Systems sozialer Kategorien für die Selbst- und Fremddefinition, Ausdrucksformen für kategoriendefinierende Merkmale und sprachliche Verfahren für die Herstellung von Kategorien im Gespräch;

- die Präferenz bestimmter Kommunikationsformen und Interaktionsmodalitäten und

- die Bevorzugung einer bestimmten Sprachästhetik und bestimmter Mittel zum Ausdruck von Geschmack (Aufmachung, Gestik und Mimik).

Die Ausprägung von Phänomenen auf diesen Ebenen und ihre Verknüpfung folgen Stilbildungsprinzipien, die zu strukturellen und ästhetischen Homologien und zum einheitlichen „Bild“ eines Gruppenstils führen. Kernfragen bei der Herausbildung eines Gruppenstils sind: Was müssen die Gruppenmitglieder tun und wie müssen sie sich verhalten, um ein echtes Mitglied zu sein? Und welche Eigenschaften müssen sie wählen, um sich klar gegen andere abzugrenzen? Wie diese Kernfragen bearbeitet und beantwortet werden können, wird an Ausschnitten aus städtischen Sozialwelten einer mittleren deutschen Großstadt gezeigt, am Beispiel von Mannheim, einer mittleren Großstadt in Baden-Württemberg. Die sozialen Welten, für die die ausgewählten Gruppen exemplarisch sind, unterscheiden sich ganz erheblich bezüglich ihrer ökonomischen, räumlichen und sozialen Lebensverhältnisse, die zu unterschiedlichen Ausprägungen von Handlungsspielräumen, sozialen Orientierungen und Ausdruckssystemen führen.

\section{Der kommunikative soziale Stil einer Frauen- gruppe aus der „Welt der kleinen Leute“ im Kontrast zu Frauen aus dem Bildungsbürgertum.}

\subsection{Die „Bastelgruppe“ (Keim 1995a und b)}

Die ethnografische Beschreibung eines traditionellen Arbeiterstadtteils (volkstümlicher Name: „die Filsbach“) von Mannheim ergab eine hochgradig ausdifferenzierte 
Wohnbevölkerung, segregiert nach sozioökonomischen und ethnisch-kulturellen Merkmalen. Durch langjährige Sanierungsmaßnahmen wurde die angestammte Bevölkerung erheblich ausgedünnt und fühlte sich durch Zuwanderer an den Rand gedrängt. Ein Teil der Stammbevölkerung arbeitete intensiv an der Aufrechterhaltung und in Auseinandersetzung mit Neueinflüssen an der Weiterentwicklung traditioneller Formen sozialer Organisation und Kultur. Exemplarisch für diese soziale Welt der angestammten Bevölkerung ist die „Bastelgruppe“, eine seit Jahren stabile Freizeitgruppe von Frauen aus dem Arbeiter- und Handwerkermilieu des Stadtteils. Die Frauen treffen sich wöchentlich in den Räumen eines gemeinnützigen Vereins zur Unterhaltung und zu Handarbeiten. Als Angehörige der Welt der „kleinen Leute“ leben sie räumlich, ökonomisch und sozial innerhalb enger Grenzen; ihr Leben ist geprägt von der Anstrengung trotz widriger Umstände „anständig“ durchs Leben zu kommen. Die Lebensbewältigung wird durch ein rigides Normen- und Wertesystem gesichert, d. h.: jede/r weiß, was sie/er zu tun hat, und was sich gehört. Zu den wichtigsten Aufgaben der Frauen gehört der Kampf gegen Armut, Auflösung der Familie und sozialen Abstieg in Arbeitslosigkeit und Alkoholismus.

In der Gruppe gibt es ein hohes Bedürfnis nach gesicherter Gemeinsamkeit hinsichtlich sozialer Kategorien und moralischer Werte und eine ausgeprägte Konsensorientierung. Verbunden damit ist eine starke soziale Kontrolle untereinander und in Bezug auf das nahe soziale Umfeld, dessen Veränderungen sehr genau beobachtet werden. Die räumliche Enge führt zu einer hohen Transparenz privater Verhältnisse, und die soziale Kontrolle zeigt sich im besonderen Interesse an der Entlarvung falscher Vornehmheit oder dem Aufdecken von verwerflichem Verhalten.

\section{a) Der Umgang miteinander}

Im Vertrauen auf die allgemeine Anerkennung von sozialen Werten und Orientierungen sind weite Bereiche des Umgangs miteinander von vorsichtig tastenden, den möglichen Verhaltensspielraum auslotenden Aktivitäten befreit. Mit den geringen Ressourcen hängt eine Orientierung auf den eigenen Handlungsspielraum zusammen: Jede sorgt zunächst für sich. Bitten und Aufforderungen sind ohne Höflichkeitsformeln und direkt formuliert, wenn sich die Sprecherin sicher ist, dass die Adressierte die Bitte/Aufforderung erfüllen kann; sie sind indirekt formuliert, wenn der Eingriff ins Territorium der Adressierten für die Sprecherin nicht genau kalkulierbar ist. Die Frauen präferieren leistungsbezogene Komplimente, durch Bezeichnungen wie „gut“, „schön“ eher nebenbei erledigt. Da auffallende Kleidung oder äußere Aufmachung als Ausdruck für Verschwendung oder des über-die-Verhältnisse-Lebens gelten, lösen solche Komplimente eher Verlegenheit aus und die Adressierte reagiert durch Herabsetzung des Gelobten.

Für Verhaltenskritik gibt es je nach Anlass unterschiedliche Verfahren. Ist der Konfliktanlass gering, werden kritische Nebenbemerkungen (unadressiert und leise gesprochen) oder spielerische Frotzelangriffe bevorzugt, auf die die Kritisierte spielerisch reagieren kann. Gewichtigere Konfliktanlässe werden durch derbe Ordnungs- 
rufe (z. B. hal=die gusch) und Drohformeln (z. B. aaschloch geb doch ruh) bearbeitet, zu offenem Streit kommt es sehr selten. Bei ernsten Problemen wird das Verhalten der Kritisierten in ihrer Abwesenheit detailliert dargestellt und schonungslos verurteilt, wobei die Gruppe davon ausgeht, dass die Kritisierte von der Kritik erfährt und ihr Verhalten stillschweigend ändert. Die subtilste Form der Kritik bildet eine besondere Form des Klatsches. Dabei wird an einer gruppenexternen Person ein Fehlverhalten dargestellt und verurteilt, das identisch mit dem des anwesenden Gruppenmitglieds ist, auf das die Kritik zielt. Aufgabe der Kritikerin ist es, die Adressatin in die KlatschInteraktion einzubeziehen und sie bei der Negativbeurteilung zu beteiligen. Auf diese Weise wird die Adressierte interaktiv dazu gebracht, sich von der kritisierten Handlungsweise zu distanzieren.

Zu den präferierten Spielformen in der Gruppe gehören Witze mit sexueller und skatologischer Thematik, vor allem „harte“ bzw. „dreckige“ Witze. Treten sie in Serie auf, findet die Gruppe zur Hochform von Geselligkeit, wie das langanhaltende, kreischende Lachen und begeisterte Anerkennung zeigen. Ähnliches Vergnügen bereiten frotzelnde Phantasiespiele, die aus mehreren Spielzügen mit Angriffen und gesteigerten Reaktionen des Opfers bestehen. Die Angriffe zielen auf weit übertriebene Eigenschaften des Opfers, sie verletzen nicht, und Angreiferin und Opfer arbeiten kooperativ auf eine drastische, komisch-groteske Pointe hin. Die Lust an der Obszönität und die kreischende Ausgelassenheit sind ein markantes sozialstilistisches Merkmal zur Abgrenzung nach außen, vor allem zu Angehörigen von „gutbürgerlichen“ Gruppen. In Gegenwart von Außenstehenden werden obszöne Witze und Phantasiespiele in spielerischer Weise selbst zensiert.

b) Sprechen über sich und andere

Beim Sprechen über sich und die Familie gilt in der Gruppenöffentlichkeit das Tabu der ernsten Thematisierung von persönlichen Problemen. Wenn Probleme in der Gruppenöffentlichkeit thematisiert werden, dann durch witzige oder grotesk-übertreibende Formen, bei denen die Betroffene mitarbeitet. Durch diese Bearbeitungsmodalität schützen die Frauen die Betroffene vor emotionaler Entblößung, die mit der ernsten Thematisierung potentiell verbunden ist.

Das Sprechen über Personen aus dem nahen sozialen Umfeld im Klatsch und „Tratsch“ (so die Bezeichnung der Frauen) gehört zu den wichtigsten kommunikativen Ereignissen in der Gruppe. Dabei fällt einerseits die Lust am Ausleben von Ärger, Empörung und Spott auf, andererseits das große Streben nach Übereinstimmung in der moralischen Bewertung. Der Neuigkeitswert einer Information ist nicht konstitutiv für ein Klatschereignis; skandalöse Verhaltensweisen von Personen können auch mehrfach bearbeitet und immer weiter expandiert und präzisiert werden. Für die moralische Beurteilung von Personen haben die Frauen ein ausdifferenziertes Kategoriensystem entwickelt, das den spezifisch weiblichen Blick auf das eigene Leben und das nahe soziale Umfeld zeigt. Status- und Rollenkategorien werden von moralischen Kategorien überlagert und in positive und negative Ausprägungen 
entlang der Werte „Anständigkeit“, "Vertrauenswürdigkeit“, „Großzügigkeit“ und „Aufrichtigkeit“ gegliedert. Mit Statuskategorien gliedern die Frauen die soziale Umwelt in Relation zu sich selbst. Den mittleren Bereich der Sozialhierarchie besetzen sie selbst als „gesunde Filsbacherinnen“. Den oberen Bereich bilden Personen mit besserer Bildung und besseren finanziellen Verhältnissen. Je nach moralischer Bewertung gibt es für diese Hierarchieebene die „Anständigen“, die sich den Frauen gegenüber respektvoll und rücksichtsvoll verhalten; die „Unverschämten“, die sich ihnen gegenüber dünkelhaft und verletzend verhalten und die „Dreckigen“, die trotz besserer Lebensbedingungen korrupt und verkommen sind. Für den unteren sozialen Bereich gibt es Kategorien, die durch Sucht, finanzielle Unselbständigkeit und durch Auflösung sozialer Bindungen charakterisiert sind. Sie werden meist negativ bewertet; doch es gibt auch positive Fälle, z. B. den „netten Penner“, der sich für sein rücksichtsloses Verhalten mit einem kleinen Geschenk entschuldigt; oder den „hilfsbereiten Alkoholiker“, der den Frauen bei schweren Hausarbeiten hilft. Angehörige beider Kategorien können mit Unterstützung und Fürsorge rechnen.

Rollenkategorien beziehen sich zum einen auf das Lebensalter und auf angemessene bzw. unangemessene Verhaltensweisen für „Junge“ und „Alte“, zum anderen auf den familiären Bereich. Das familiäre Kategoriensystem basiert auf der traditionellen ehelichen Normalform, in der das Rollenverhältnis zwischen Ehemann und Ehefrau durch Aufgaben und Pflichten geregelt ist: Der Mann sorgt für den Familienunterhalt, die Frau versorgt den Haushalt und erzieht die Kinder. Die Rolle des Mannes ist in der Familie dominant, Frau und Kinder richten sich nach seinen Bedürfnissen. Beide Ehepartner verfolgen getrennte Freizeitaktivitäten, der Mann in den Stadtteilwirtschaften, die Frau mit anderen Frauen. Neben den Kategorienbezeichnungen für Ehepartner, „Mann“ und „Frau“, die der ehelichen Normalform entsprechen, sind Kategorien von besonderem Interesse, für die abweichende Dominanzverhältnisse oder Devianzmerkmale charakteristisch sind. Da gib es z. B. den „Hausdrachen“, eine Ehefrau, die ihren Mann in allen Lebensbereichen kontrolliert, und den zu ihr passenden „Hampelmann“, der sich dem Kontrollanspruch seiner Frau fügt. Das männliche Gegenstück zum „Hampelmann“ ist der „Pascha“, der sich in die Aufgaben der Frau einmischt und sie kontrolliert; zu ihm gehört die „dumme Frau“, die sich seinen Ansprüchen fügt. Eine für die Frauen interessante Kategorie ist die „couragierte“ Frau, die sich den Ansprüchen des „Paschas“ widersetzt; sie wird von den Frauen positiv bewertet.

c) Sprachvariation und formelhaftes Sprechen

Neben gesprächsorganisatorischen Funktionen (z. B. bei Fokussierungen, bei Reparaturverfahren und beim Kampf ums Rederecht) dient die Variation zwischen standardnahen und dialektalen Formen zum symbolischen Verweis auf soziale Kategorien. Präferierte Strukturteile sind Zitate oder Quasizitate, eigene Handlungsalternativen zu negativ bewerteten Handlungen anderer und explizite Bewertungen. Die Bedeutung von Standard und Dialekt ist dabei variabel, an die Beurteilung von Personen 
und deren Handlungsweisen gekoppelt und an die Zuordnung zu negativ oder positiv bewerteten Kategorien. Standard wird z. B. mit positiver Bewertung verbunden, wenn auf einen allgemeinen Rechts- und Moralkodex verwiesen wird oder auf eine Person, die sich für die Belange der Frauen einsetzt. Standard ist mit negativer Bewertung verbunden, wenn auf Statushöhere verwiesen wird, die sich den Beteiligten gegenüber arrogant und abweisend verhalten. Auf das Verhalten angeblich vornehmer Leute, die sich durch unangemessenes Verhalten selbst entlarven, wird mit Spott und übertriebenem, affektiertem Standard verwiesen. Breiter Mannheimer Dialekt kann positiv bewertet werden, wenn Statushöhere sich demonstrativ mit den Frauen solidarisieren; oder zur Selbstreferenz, wenn die Beteiligten auf die Distanzierung von Statushöheren derb reagieren. Dialekt wird mit negativer Bewertung verbunden, wenn auf grobes, rücksichtsloses und gewalttätiges Verhalten verwiesen wird.

Zum Ausdrucksrepertoire gehören auch Formeln wie Redewendungen, Sprichwörter, Gemeinplätze, Formeln mit sozial-geografischem Bezug und gruppenspezifische Formeln. Formeln sind konstitutiv für die Beziehungsregulierung, für Begrüßungen und Verabschiedungen, für den Hinweis auf Regelverstöße und für spielerische oder aggressive Frotzel-Interaktionen. Neben der Variation in Sprache und Sprechweise spielen Formeln vor allem im Prozess der sozialen Kategorisierung eine bedeutende Rolle. Die Gruppenmitglieder wissen, welcher Typ von Formeln zu welchen Zwecken verwendet wird. In dem gemeinsam durchgeführten Prozess der sozialen Kategorisierung z. B., in dem die erste Phase eine dem aktuellen Geschehen nahe Schilderung eines skandalösen Ereignisses enthält, können allgemeine Phraseologismen zur Charakterisierung der handelnden Person vorkommen. In der zweiten Phase erfolgen zugespitzte Reformulierungen des Dargestellten. In dieser Phase entstehen die gruppenspezifischen Formeln wie z. B. er muss häm die Bedde mache, sie geht in die Lokale zur Charakterisierung der Beziehung zwischen dem übermächtigen „Hausdrachen“ und dem gefügigen „Hampelmann“. In der dritten Phase erfolgt die Generalisierung des Falles durch Sprichwörter, Gemeinplätze oder Maximen, in denen allgemeine Erfahrungen gebunden sind, z. B. „wo die Liebe hinfällt“. In der letzten Phase wird dann der Kategorienname genannt.

Der virtuose Wechsel in Sprache und Sprechweise und der virtuose Einsatz verschiedener Formeltypen sind konstitutiv für gemeinsam durchgeführte Prozesse sozialer Kategorisierung. Auf diese Weise zeigen sich die Beteiligten wechselseitig, dass sie in ihrer eigenen Welt sind; Nicht-Filsbacher sind davon ausgeschlossen.

\subsection{Eine Frauengruppe aus der Welt des Bildungsbürgertums, die „Literaturfrauen“ (Schwitalla 1995a und b)}

Die Frauen der Literaturgruppe, ebenfalls eine Freizeitgruppe, gehören zur tonangebenden Schicht eines in den 60er und 70er Jahren des letzten Jhds. in Mannheim neu angelegten Stadtteils. Sie selbst und/oder ihre Männer üben dort in Kirche, Politik, 
Sport und Kunst organisatorische Tätigkeiten aus und sind im Stadtteil gut vernetzt. Die Frauen sind mittleren bzw. höheren Alters, die meisten verheiratet mit erwachsenen Kindern. Es sind vor allem Hausfrauen, einige arbeiten in akademischen Berufen. Die Frauen haben einen weiten Bekannten- und Freundeskreis und eine hohe überregionale Mobilität. Sie sind vertraut mit dem Wohn- und Lebensstil der übrigen Mitglieder der Gruppe und verbringen auch gemeinsame Urlaube oder unternehmen Theater- und Ausstellungsbesuche. Das offizielle Ziel der Treffen sind Lesungen anspruchsvoller Literatur und das Besprechen des Gelesenen; das inoffizielle Ziel jedoch ist der Austausch von Informationen und die Erörterung persönlicher Probleme. Die Gruppentreffen finden regelmäßig in der Wohnung einer der Frauen statt.

\section{a) Umgang miteinander}

Große sozialstilistische Unterschiede zwischen den beiden Frauengruppen liegen im Bereich des Umgangs miteinander (vgl. auch Kallmeyer 1995b). Die Literaturfrauen verfügen in selbstverständlicher Weise über einen großen ökonomischen und sozialen Handlungsspielraum. Ihre Leitvorstellung ist: „Wir lieben das Schöne, Gebildete und gehen vorsichtig miteinander um“. Ein wichtiges Prinzip ist der besondere Schutz der Privatsphäre der anderen; jede hat ein großes Territorium und respektiert das der anderen. Auch kleine Eingriffe ins Territorium der andern, z. B. bei Bitten und Aufforderungen werden mithilfe konventioneller Höflichkeitsformen deutlich abgeschwächt. Die Frauen ignorieren kleine Fehlgriffe, loben positive Aspekte und versuchen negative Gefühle möglichst zu vermeiden.

Die besondere Aufwertung positiver Eigenschaften anderer ist charakteristisch für die Gruppe. Positiv gewertete Eigenschaften, z. B. schöne Kleidung und schönes Aussehen, werden lobend hervorgehoben und in expressiver und stilistisch variierender Weise bearbeitet. Die emphatische Betonung der Wertschätzung der anderen, der expressive Ausdruck positiver Gefühle zueinander bei gleichzeitiger Kontrolle negativer Gefühle (Ärger, Wut, Zorn), der ausdrucksstarke Mitvollzug von Gefühlen Betroffener schafft die Basis für eine persönliche Nähe, ohne dass das Selbstbestimmungsrecht der Einzelnen tangiert wird.

Lachen ist eher zurückhaltend und gedämpft im Vergleich zum langanhaltenden Kreischen der Filsbachfrauen. Das Zeigen oder gar Ausleben negativer Gefühle wird vermieden, eine ablehnende Einstellung zu Handlungen anderer wird indirekt oder ironisch ausgedrückt. Wenn Konflikte auftreten, besteht die Präferenz für explizite Thematisierung und Argumentation. Wenn dabei spontan geäußerte negative Bewertungen auftreten, werden sie sofort zurückgenommen.

b) Sprechen über sich und andere

Ein weiterer kommunikativer Bereich, in dem große sozialstilistische Unterschiede zwischen den beiden Gruppen bestehen, ist das Sprechen über sich und andere. Im Gegensatz zu den Frauen der Bastelgruppe sprechen die Literaturfrauen auch in der Gruppe offen, ausführlich und in ernster Modalität über persönliche Probleme. Wenn 
eine Betroffene ihr Problem thematisiert, wird sie von den anderen zum offenen Sprechen eingeladen. Dabei zeigen die Frauen große Empathie und bieten sich als Helferinnen an. Beim Sprechen über sich und die Familie sind familiäre Rollenkategorien von Bedeutung, die die Eigenschaften für die „Ehefrau“ und „Mutter“ festlegen. Das Schicksal der erwachsenen Kinder und Probleme mit den Kindern werden ausgiebig und mit großem Engagement besprochen. Dabei kommt immer wieder die eigene Mutterrolle in den Blick. Ereignisse und Handlungsweisen, die die Ehefrauen- und Mutterrolle betreffen, können argumentativ behandelt werden, da es in der Gruppe unterschiedliche Auffassungen und lebenspraktische Lösungen gibt. Doch sobald eine der Frauen persönlich stark betroffen ist, gehen die anderen sehr behutsam mit ihr um.

Beim Sprechen über familiäre Konflikte stellen die Frauen die Modalität der Themenbehandlung darauf ab, ob das Problem gewichtig oder gering ist und ob es neu oder bereits bewältigt ist. Kleine, bewältigte Probleme werden bevorzugt ironisch und scherzhaft behandelt. Bei unbewältigten Problemen lassen die Frauen, wenn die Betroffene es will, lange, ernste und von Empathie getragene Gespräche zu. Die Frauen sprechen auch ausgiebig über moralische Bewertungskriterien und behandeln unterschiedliche Perspektiven reflexiv und argumentativ. Am Ende der Diskussion besteht kein Zwang zum Konsens. Wichtig ist jedoch die Meinung der anderen zu verstehen, ihren Problemen gegenüber Empathie zu zeigen, ihnen zu zeigen, dass man mit- und nachempfinden kann, auch wenn man anderer Meinung ist.

Im Gegensatz zur Bedeutung des Klatsches in der Bastelgruppe spielt das Sprechen über Personen, welche die Frauen aus ihren Stadtteilengagements kennen, kaum eine Rolle; die soziale Abgrenzung zu anderen sozialen Gruppen ist für sie nicht relevant.

c) Sprachliche Präferenzen und Vermeidungen

Auch in diesem Bereich bestehen große Unterschiede zur Bastelgruppe. Die Literaturgruppe präferiert die Normalform der Standardsprache, der Wechsel in dialektale o. a. andere Sprechweisen kommt kaum vor. In der Gruppe wird vulgäre, grobe und aggressive Lexik vermieden; die Frauen verwenden gelegentlich auch literarische Ausdrucksweisen (z. B. sich entäußern, echauffiertsein, Schwitalla 1995a, 281). Sprichwörter oder formelhafte Wendungen kommen kaum vor. Es werden keine sexuellen Themen behandelt, Gewalt oder andere Formen sozialer Devianz kommen nicht vor. Bei vielen Themen lassen die Frauen verschiedene Meinungen zu, individuelle Sichtweisen werden gegeneinander abgewogen und diskutiert. Es besteht kein Druck zur Herstellung von Beurteilungsgemeinsamkeit, und für Alltagsprobleme gibt es keine gültigen Lösungsverfahren, die mit allgemeingültigen Formeln und Sprichwörtern u. a. wechselseitig bestätigt werden.

Ein großer sozialstilistischer Unterschied zwischen beiden Gruppen besteht bei der demonstrativen Herstellung von Gemeinsamkeit. Die Bastelgruppe präferiert dafür Klatschkommunikation verbunden mit starker Expression von Wut und Empö- 
rung; und beim Erzählen „dreckiger“ Witze wird Gemeinsamkeit geradezu zelebriert, ausgedrückt durch langanhaltende, kreischende Ausgelassenheit. Bei den Literaturfrauen dagegen spielen Formen des ironischen Sprechens eine große Rolle, und für die Herstellung einer Atmosphäre der ,emotionalen Wärme und des gegenseitigen Vertrauens“ (a.a. O., 237) sind hyperbolische Expressivität, emphatisches Komplimente-Machen und deutliches Zeigen von Empathie und Mitgefühl bei der Behandlung persönlicher Probleme charakteristisch.

Die wesentlichen Stilmerkmale der beiden Gruppen können auch in anderen sozialen Welten beobachtet werden. Die Verhaltensweisen der Literaturgruppe sind charakteristisch für Gruppen des Bildungsbürgertums und der Intellektuellen (a.a. O., 237); Verhaltensweisen der Bastelgruppe mit der Präferenz für die offene Darstellung von Körperlichkeit, für Derbheit und ausgelassene Entgrenzung werden in der soziologischen Literatur vielfach beschrieben ( z. B. Bourdieu 1982 und Elias 1995); sie sind auch charakteristisch für volkstümliche Formen der Unterhaltung im Schwank und im Volks- und Bauerntheater.

\section{Der kommunikative soziale Stil der „türkischen Powergirls“ und seine Veränderung im Laufe der Zeit (Keim 2008 und 2012)}

Auf der Grundlage der ethnografischen Beschreibungen der Lebenswirklichkeit junger türkischstämmiger MigrantInnen in Mannheim wurden drei Gruppen als Repräsentanten sozialer Welten der Migration ausgewählt, die sich aus den ethnischkulturellen Traditionen und normativen Orientierungen der Migrantengemeinschaft lösen und sich in wichtigen Lebensbereichen mit der Mehrheitsgesellschaft produktiv auseinandersetzen: die „Europatürken“ (Aslan 2005), die emanzipatorischen Migranten (Cindark 2010) und eine Gruppe junger Frauen auf dem Weg zur Emanzipation, die „türkischen Powergirls“ (Keim 2008). Die genannten Gruppen unterschieden sich in ihren sozialen Orientierungen, ihrem Selbstbild und ihrem Ausdrucksverhalten ganz erheblich. Ein ausführlicher Vergleich der kommunikativen Stile der „Europatürken“ und der „emanzipatorischen Migranten“ findet sich in Cindark (2010).

Im Folgenden werde ich die „türkischen Powergirls“ vorstellen, eine Gruppe junger Frauen, deren mehrjährige ethnografische Beobachtung es ermöglichte, stilistische Veränderungen zu erfassen, die im Laufe der biografischen und sozialen Entwicklung auftraten. Am Beginn der Beobachtung sind die jungen Frauen noch in der Migrantenpopulation verwurzelt, rebellieren aber gegen die Vorstellungen der Elterngeneration und arbeiten in ständiger Auseinandersetzung mit der deutschen und türkischen Umwelt an einem eigenständigen Selbstbild, das sie am Ende der Beobachtungszeit auch erreichen. Im Prozess dieser Entwicklung von der Rebellion zur 
Emanzipation und mit der erfolgreichen Teilhabe an neuen sozialen Welten verändert sich der kommunikative Stil der Gruppe. An diesem Fall zeigt sich die Beschreibungs- und Erklärungsstärke des Stil-Konzepts, da es sowohl den Zusammenhang zwischen Lebenswelt und Ausdrucksverhalten erfassen, als auch Veränderungen in der Lebenswelt mit stilistischen Veränderungen in Zusammenhang bringen kann.

a) Biografische und soziale Entwicklung

Die Gruppenmitglieder, Kinder ehemaliger „Gastarbeiter“, sind in einem Stadtteil mit hohem Migrantenanteil aufgewachsen, der sich durch ökonomische, ethnische und soziale Einheitlichkeit deutlich von anderen Stadtgebieten unterscheidet. Die Familien, die aus Mittel- und Ostanatolien kommen, streben für die Töchter eine höhere Schul- und eine gute Berufsausbildung an mit dem Ziel der finanziellen Eigenständigkeit. Die Mädchen sind gute Grundschülerinnen und die meisten schaffen den Übergang in höhere Schulen, die außerhalb des Migrantenstadtteils liegen. Dort erfahren sie zum ersten Mal sprachliche und soziale Anforderungen, auf die sie nicht vorbereitet sind. Den Übergang in diese Schulen erleben sie als Schock des Lebens; sie fühlen sich fremd, ausgelacht, ausgegrenzt und allein gelassen. Fast zeitgleich mit den schulischen Problemen beginnen auch innerfamiliäre Auseinandersetzungen. In vielen Migrantenfamilien gilt für die Erziehung von Töchtern das Leitbild der ,traditionellen jungen Türkin“, bescheiden, zurückhaltend und sich ganz den Wünschen der Familie unterordnend. Mit Beginn der Pubertät versuchen die Eltern die Mädchen nach diesem Leitbild zu erziehen; sie schränken den Lebensraum auf den häuslichen und familiären Bereich ein und kontrollieren die außerhäuslichen Kontakte. Die Mädchen rebellieren und wollen die Freiheiten durchsetzen, die sie bei anderen Jugendlichen sehen. Die Eltern regieren mit Strenge und setzen damit eine Spirale von Gewalt und Widerstand in Gang bis hin zu körperlichen Strafen. Den Konflikt mit den Eltern deuten die Mädchen als Konflikt mit den türkischen Traditionen und Leitbildern, die sie auch als Ursache für die in der Schule erlebte Ausgrenzung sehen. Die ethnische Deutung familiärer und schulischer Probleme führt zur Bildung einer ethnischen Clique als einer Art Schicksalsgemeinschaft: Hier konstruierten die Mädchen ein Selbstbild, mit dem sie dem sozialen Druck aus der Migrantengemeinschaft widerstehen und die Ausgrenzungen, die sie in der deutschen Schule erleben, verarbeiten können. Sie bezeichnen sich als „türkische Powergirls“ mit den Eigenschaften „stark“, „aggressiv“ und „cool“. Im Kontrast zu einem von deutschen Gymnasiallehrern erwarteten Schülerverhalten gebärden sie sich in der Schule grob, schroff und widerständig. Die übersteigerte Ethnizität und die antischulische Haltung setzen eine Entwicklungsdynamik in Gang, die zum Ausstieg aus beiden Bezugswelten und zu sozialer Devianz führt.

b) Stil als Ausdruck der Rebellion gegen beide Bezugswelten Die Powergirls stellen in Bezug auf folgende Ausdrucksdimensionen ein Gegenbild zu den sozialen Leitbildern her, gegen die sie sich abgrenzen. 
Geschmack und Sprachästhetik: Im Kontrast zum äußeren Erscheinungsbild der „traditionellen jungen Türkin“, die sich unauffällig und bedeckt kleidet, orientieren sich die Powergirls an einem modischen, flippigen Jugendstil. Sie kleiden sich offen, bunt und körperbetont, sind stark geschminkt und gepierct. Entgegen dem Verbot außerhäuslicher Kontakte haben sie Freunde, besuchen Lokale und Discos. Dem zurückhaltenden Verhalten der „traditionellen jungen Türkin“ setzen sie ein ungezügeltes, aufbegehrendes Verhalten entgegen und greifen dabei auf sprachliche Merkmale männlicher Jugendlicher zurück, die sie mit Grobheit, Stärke und Aggressivität verbinden. Sie verwenden derbe männliche Anredeformen, drastische Beschimpfungsformeln und praktizieren obszöne Droh- und Übertrumpfungsrituale.

Umgang miteinander: Die Formen für den Umgang miteinander und für den Ausdruck von Gefühlen vermitteln ein klares Gegenbild zu dem Leitbild der „traditionellen Türkin“, ebenso wie zu dem Idealbild einer kultivierten Gymnasiastin. Für die Powergirls ist die wechselseitige Respektierung territorialer Ansprüche wichtig, ebenso wie die erfolgreiche Verteidigung des eigenen Territoriums. Kritik ist offen, direkt und unverblümt. Übergriffe in den Privatbereich werden durch derb-drastische Beschimpfungs- und Drohformeln zurückgewiesen, die zu rituellen Beschimpfungen mit obszönen Formeln gesteigert werden können. Bei Aushandlungen wird weniger argumentiert als gestritten nach dem Grundsatz, wer am lautesten schreit und die andere am schärfsten angreift, setzt sich durch. Gegenseitiges Lob ist direkt und aufrichtig; Verstecken der eigenen Meinung hinter gefälligen Formulierungen wird als „anschleimen“ abgelehnt. In Antizipation von Einschränkung oder Ausgrenzung seitens der Erwachsenen sind die Powergirls in ständiger Abwehrbereitschaft, was stilistisch in einer besonderen Reaktionsschnelligkeit zum Ausdruck kommt, mit der Anforderungen abgewiesen, eigene Vorteile durch schnelles Zupacken gesichert und erlebte Benachteiligungen durch ungebrochene Aggression pariert werden. Negative Emotionen, Ärger, Zorn, Wut werden durch Beschimpfungsformeln, durch Schreien, aggressive Mimik und Gestik oder tätliche Angriffe ausgedrückt; positive Emotionen durch schallendes Lachen, Freudenschreie, Freudentänze oder stürmisches Umarmen. Abschwächungen, Entschuldigungen kommen in der Ingroup-Kommunikation kaum vor.

Verwendung sprachlicher Ressourcen: Die Powergirls verfügen über ein reiches sprachliches Repertoire, das sie in virtuoser Weise einsetzen. Mit Deutschen sprechen sie Umgangsdeutsch, mit Türken Umgangstürkisch. Mit Bilingualen verwenden sie deutsch-türkische Mischungen, für die sie Bezeichnungen wie „Mischmasch“, „Mixmax“ oder „Mischsprache“ haben. Die Mischsprache ist in hohem Maße durchstrukturiert. Auch wenn sie unter grammatischem Aspekt (noch) keine eigene Varietät bildet, ist sie unter soziolinguistischem Aspekt eine eigenständige Ausdrucksform, die zu dieser sozialen Welt gehört. Die innere Variabilität der Mischsprache, die Bewegungen auf einem Kontinuum zwischen Türkisch und Deutsch mit verschiedenen, relativ systematisch auftretenden Sprachwechselmustern zeigen die Arbeit der Sprecherinnen an dieser Ausdrucksform. Die Mischsprache hat für die Gruppe 
einen hohen Stellenwert; sie ist Symbol für der Gruppe und bildhaftes Zeichen für ihre sozial-kulturelle Abgrenzung zu beiden Bezugswelten. Ästhetische Performanz kommt in der Selbstverständlichkeit, Leichtigkeit und Eleganz zum Ausdruck, mit der die Mischsprache praktiziert wird.

c) Vom Stil der Rebellion zum Stil der Emanzipation: Der Weg zu sozial akzeptierten Lebenswegen und Ausdrucksweisen

Voraussetzung für diesen Weg ist das Auseinanderbrechen der ethnischen Clique und die Orientierung an einem neuen Leitmodell für soziales und kommunikatives Verhalten. Das finden die jungen Frauen in der deutsch-türkischen Leiterin einer Jugendeinrichtung, die ihnen neue Perspektiven auf die türkische und deutsche Bezugswelt eröffnet und sie bei der Entwicklung eines neuen Selbstbildes unterstützt. Die Leiterin schafft Anlässe zur Übernahme von sozialer Verantwortung, motiviert die Mädchen zum Sprechen über schmerzliche Diskriminierungserfahrungen, bricht stereotype Denk- und Verhaltensmuster auf und führt intelligente Abwehrverfahren vor. Die eigene biographische Entwicklung dient dabei als Beleg für die Wirksamkeit der gelehrten Inhalte. Die Leiterin wird zum Bezugspunkt für einsetzende Veränderungen im kommunikativen Stil der Powergirls, und den Mädchen gelingt die Entwicklung neuer Ausdrucksformen, die in den Bildungsinstitutionen akzeptiert sind. Der Erwerb neuer stilistischer Ausdrucksmittel bedeutet jedoch nicht, dass alte aufgegeben werden. Es gibt Phasen im Veränderungsprozess, in denen konträre Stilelemente koexistieren und zum Verweis auf unterschiedliche Kontexte eingesetzt werden. Und es gibt Phasen, in denen die Inkorporation neuer stilistischer Elemente zunächst mit Distanzierungsmarkierungen, dann ohne Distanzierung beobachtbar ist. Jedes neue stilistische Element wird auf seine Passung und rhetorische Wirksamkeit hin überprüft, aufgenommen oder (noch) verworfen. Charakteristisch für das stilistische Repertoire ist die Gegensätzlichkeit der stilistischen Mittel, zwischen denen die Sprecherinnen in virtuoser Weise wechseln: Auf drastische und obszöne Formen, die in schneller Reaktion auf derbes Verhalten verwendet werden, können elaborierte Formen folgen, mit denen die Sprecherinnen eine neue Situation schaffen und sich als kultivierte Gesprächspartnerinnen präsentieren. Der Stil der ehemaligen Powergirls verändert sich von einem „Stil der Rebellion“ hin zu einem „Stil der Emanzipation“ in Bezug auf die folgenden Ausdrucksdimensionen.

Geschmack und Sprachästhetik: Es gibt zunehmend Routinen mit konventionellen Höflichkeitsformeln und die Akzeptanz derb-drastischer Formeln verschiebt sich. Die Gruppenmitglieder behalten zwar ihre Kompetenz in groben, obszönen Ausdrucksweisen, doch sie haben gelernt zwischen Situationen zu unterscheiden, in denen sie wirksam sind, und Situationen, in denen sie unangemessen sind.

Umgang miteinander: Der Umgangston untereinander wird höflicher, die Gruppenmitglieder beginnen Rederechtsregeln zu respektieren, sich bei Übergriffen $\mathrm{zu}$ entschuldigen und den Ausbruch von Emotionen zu kontrollieren. Vor allem hat sich das stilistische Bewusstsein geschärft: Die Gruppe beginnt den typischen 
„Powergirl“-Stil kritisch zu betrachten, selbstironisch zu kommentieren und Distanz dazu aufzubauen.

System sozialer Kategorien und die Verwendung sprachlicher Ressourcen: Deutsch nimmt in vielen Lebensbereichen zu und deutsch-türkische Mischungen werden auf bestimmte Genres (z. B. Erzählungen) und auf den privaten Austausch reduziert. Mischungen bleiben aber das zentrale Kommunikationsmittel im privaten Bereich.

Das Selbstbild, das die jungen Frauen entwickeln, ermöglicht es ihnen, sich dem gegenläufigen Anpassungsdruck aus beiden Bezugswelten zu entziehen. Sie definieren sich jenseits der ethnischen Kategorien „türkisch“ und „deutsch“ als etwas „Neues“, als „Deutsch-Türkin“. Das verändert auch den Blick auf die umgebende Lebenswelt, und die jungen Frauen entwickeln ein System sozialer Kategorien, das das Ergebnis ihrer Analyse des sozialen Umfeldes und seine Bewertung zum Ausdruck bringt. Dieses Kategoriensystem ist in zwei Sets unterteilt, eines für die Welt der Deutschen und ein wesentlich weiter ausdifferenziertes für die Welt der türkischen Gemeinschaft; das deutet darauf hin, dass die jungen Frauen mit der türkischen Gemeinschaft mehr Problembereiche zu bewältigen haben, als mit Deutschen. In Bezug auf die türkische Gemeinschaft haben zwei Negativkategorien hohe Priorität: zum einen der „zurückgebliebene Türke“, eine Kategorie für integrationsunwillige Angehörige der Elterngenerationen, und zum anderen die „asoziale Türkin“ und ihr männliches Pendant, der „türkische Macho“, traditionell orientierte Angehörige der zweiten/dritten Migrantengeneration. In Bezug auf die Welt der Deutschen sind zwei Kategorien relevant, deren Angehörige sich den jungen Frauen gegenüber ausgrenzend verhalten: der „gestörte Lehrer“ und der „dumme Deutsche“

Im Zusammenhang mit sozialer Kategorisierung haben sich rhetorisch besonders wirksame Verfahren zum Verweis auf soziale Kategorien und Kontexte herausgebildet. Dazu werden die Deutschvarietäten „Gastarbeiterdeutsch“, das rudimentäre Deutsch von Angehörigen der Elterngeneration, „Mannheimerisch“ und ethnolektal geprägtes „Ghettodeutsch“ verwendet (Keim 2012, Kap. 5). Zwischen Sprache und sozialer Kategorie gibt es auch hier keine einfache Zuordnung, sondern je nach Gesprächskontext, Adressatin und Perspektivierung können diese Varietäten verschiedene Funktionen ausfüllen. Gastarbeiterdeutsch z. B. wird zur Symbolisierung der negativen Kategorie des „zurückgebliebenen Türken“ verwendet; im Gespräch mit Deutschen kann Gastarbeiterdeutsch verwendet werden, um die Gesprächspartner mit einem stereotypen Bild von Migranten zu konfrontieren, zu provozieren und zur Stellungnahme zu zwingen. Mannheimerisch kann zur Kontextualisierung von Blödelei und Spiel, aber auch zur Symbolisierung des „dummen Deutschen“ eingesetzt werden; dazu gehören Deutsche, die die jungen Frauen als schlicht und grob erlebt haben, und die sich ihnen gegenüber feindselig verhalten.

Am Ende der Beobachtungszeit haben die jungen Frauen erfolgreich die höheren Schulen abgeschlossen und eine Ausbildung oder einen akademischen Abschluss erreicht. Ihr Selbstbild jenseits ethnischer Kategorien als „Deutsch-Türkin“ hat sich 
stabilisiert. Als Symbol dafür fungiert die Mischsprache, die sie auch zum ausschlaggebenden Kriterium für die Partnerwahl machen: Als Partner kommen nur Personen in Frage, die ebenfalls Mischsprache sprechen.

\section{Literatur}

Aslan, Sema (2005): Aspekte des kommunikativen Stils einer Gruppe weltläufiger MigrantInnen türkischer Herkunft: Die „Europatürken“. In: Deutsche Sprache 4/2004, 327-356.

Bourdieu, Pierre (1982): Die feinen Unterschiede. Kritik der gesellschaftlichen Urteilskraft. Frankfurt a. M.

Cindark, Ibrahim (2010): Migration, Sprache und Rassismus. Der kommunikative Sozialstil der „Unmündigen“ als Fallstudie für die „emanzipatorischen Migranten“. Tübingen.

Clarke, John u. a. (1979): Jugendkultur im Widerstand. Milieus, Rituale, Provokationen. Frankfurt a. $M$.

Cressey, Paul G. (1932): The Taxi-dance Hall. A Sociological Study in Commercialized Recreation and City Life. Chicago. Neuauflage 1969.

Gumperz, John/Dell Hymes (Hg.) (1964): The ethnography of communication. In: American Anthropologist 66/6, Pt.2

Gumperz, John/Dell Hymes (Hg.) (1972): Directions in Sociolinguistics. The Ethnography of Communication. New York.

Heath, Shirley Bride (1983): Ways with Words. Language, Life, and Work in Communities and Classrooms. Cambridge.

Irvine, Judith (2001): „Style“ as distinctiveness: culture and ideology of linguistic differentiation. In: Penelope Eckert/John Rickford (Hg.): Style and Sociolinguistic Variation. Cambridge, 21-43.

Kallmeyer, Werner (Hg.) (1995): Kommunikation in der Stadt. Teil 2, Ethnographien von Mannheimer Stadtteilen. Berlin.

Kallmeyer, Werner (1995): Ethnographie städtischen Lebens. Zur Einführung in die Stadtteilbeschreibungen. In: Werner Kallmeyer (Hg.), 1-41.

Kallmeyer, Werner (1995): Zur Darstellung von kommunikativem sozialem Stil in soziolinguistischen Gruppenporträts. In: Inken Keim (1995) 1-25.

Keim, Inken (1995): Kommunikative Stilistik einer sozialen Welt „kleiner Leute“ in der Mannheimer Innenstadt. Kommunikation in der Stadt. Teil 3. Berlin.

Keim, Inken (1995): Die Westliche Unterstadt. In: Werner Kallmeyer (Hg.), 42-188.

Keim, Inken (2008): Die „türkischen Powergirls“. Lebenswelt und kommunikativer Stil einer Migrantinnengruppe in Mannheim. 2. Aufl. Tübingen.

Keim, Inken (2012): Mehrsprachige Lebenswelten. Sprechen und Schreiben der türkischstämmigen Kinder und Jugendlichen. Tübingen.

Schütze, Fritz (2002): Das Konzept der sozialen Welt im symbolischen Interaktionismus und die Wissensorganisation in modernen Komplexgesellschaften. In: Inken Keim/Wilfried Schütte (Hg.): Soziale Welten und kommunikative Stile. Festschrift für Werner Kallmeyer zum 60. Geburtstag. Tübingen, 57-83.

Schwitalla, Johannes (1995): Die soziale Welt des gebildeten Bürgertums. In: Johannes Schwitalla: Kommunikative Stilistik zweier sozialer Welten in Mannheim-Vogelstang. Kommunikation in der Stadt, Teil 4. Berlin, 3-297.

Schwitalla, Johannes (1995): Vogelstang. In: Werner Kallmeyer (Hg.), 189-343. 
Soeffner, Hans-Georg (1986): Stil und Stilisierung. Punk oder die Überhöhung des Alltags. In: Hans-Ulrich Gumprecht/Ludwig Pfeiffer (Hg.): Stil. Geschichten und Funktionen eines kulturwissenschaftlichen Diskurselements. Frankfurt a. M., 317-341.

Strauss, Anselm (1984): Social worlds and their segmentation. In: Norman Denzin (Hg.): Studies in Symbolic Interaction. Bd. 5. Greenwich, 123-139.

Suttles, Gerald (1968): The Social Order of the Slum. Ethnicity and Territory in the Inner City. Chicago.

Whyte, William Foote (1943): Street Corner Society. The Social Structure of an Italian Slum. Chicago. Willis, Paul E. (1981): „Profane Culture“. Rocker, Hippies: Subversive Stile der Jugendkultur. Frankfurt a. M. 